\title{
Modeling Iced Bio-Bandage Design for Skin Burns
}

\author{
Arar Alkhader ${ }^{1}$, Junling $\mathrm{Hu}^{2}$, Akinwumi Akinkunmi ${ }^{1}$, Prabir Patra ${ }^{1}$, Xingguo Xiong ${ }^{3}$ \\ 1 Department of Biomedical Engineering, University of Bridgeprot, Bridgeport, CT, USA \\ 2 Department of Mechanical Engineering, University of Bridgeprot, Bridgeport, CT, USA \\ 3 Department of Electrical Engineering and Computer Engineering, University of Bridgeprot, Bridgeport, CT, USA
}

\begin{abstract}
Over the years, many designs for biobandage were introduced for different types of burns but in most cases these designs are introduced as a protection means to cover and protect the burned tissue from the bacterial infection not as a treatment means. In this paper a new model for a burns biobandage is introduced not only as a protective means but also as a treatment technique by helping the tissue to rebuild itself as fast as possible. The main objective of this research is to develop a simple cryotherapeutic system to reduce the temperature of the burned tissues to normal temperature of the human body in order to facilitate the tissue healing and regeneration process. $A$ biobandage is proposed to include an iced layer as a cooling source, a cotton layer and a water gel layer for comfort and temperature control, and a plastic layer to seal the ice layer. The optimal combination of these four layers physically work together to reduce inflammation, which in turn makes the heeling or recovery time shorter and reduces pain as a result of decreasing the nerve conductivity. The COMSOL Multiphysics was used to model the cooling process on burned tissue using the proposed iced-biobandage. The calculated temperature profiles along the depth biobandage and burned skin provide a clear vision of the heat flow within each layers. The history of temperature and heat transfer rate at the burned skin surface are monitored for an effective cooling and healing process. A modeling analysis was performed to examine the changes of temperature over a predetermined time and to help in identifying the optimal period for ice cooling process, the analysis shows that the ice layer is effective within a certain period of time and after this period it doesn't add any beneficial effect.
\end{abstract}

Keywords-Heat transfer; simulation; phase change; biobandage

\section{INTRODUCTION}

Skin is mainly composed of three layers, the external layer which is called epidermis, the second layer (under-layer) is dermis which is thicker and contains hair follicles, sweet glands, and nerve ending for sensation and feeling pain. The third and the deepest layer is the subcutaneous layer, this layer is a tissue of fat that helps in maintaining the body temperature, and in other words it acts as insulator. Muscles and bones are underneath all these layers.

There are many reasons that causes damage to skin and tissues like diseases and accidents, burns are one of the most common accidents which vary in types and depth. The type of accident refers to causes like electrical, chemical, etc., While depth of burns reflects the degree; it depends on the temperature and contact duration with the burning substance. There are four major degrees of burns, first, second, third, and fourth degree burns. Greater degree means greater damaged tissue, and more complicated situation and treatment procedure.

Ice is one of the simplest and cheapest cooling techniques; usually the person after any simple burn (first and second degree burns) tries to cool the burned part with cold water or ice to reduce pain and swelling. This method of cooling are considered as a type of cryotherapeutic treatment.

Physically, the purpose of ice is to manage the heat production of the burned tissue by reducing its temperature, this reduces the blood flow demand (blood perfusion) and decreases the metabolic rate of the damaged cells [1]. Lowering the temperature makes the heeling or recovery time shorter and decreases the nerve conductivity which in turn reduces pain as well.

So, the benefits of icing can be gained by applying it in different ways, the suggested way in this project is to be as an additional layer with a combination of other different layers in a biobandage design, but these simple techniques have an intrinsic flow representing in that the temperature of the ice pack rises over time of use.

There are many commercial devices that overcome this problem, like Cryo-Cuff which contains a cooling liquid circulation system to maintain external temperature, but these devices are considered costly compared to a simple ice pack.

\section{BASIC CONCEPTS OF THE MODEL}

\section{A. Icing Protocol}

There are many diverging opinions on the most effective way of topical cooling and the best standard protocol which includes disagreements about using intermitted or continuous cooling, the optimal cooling temperature and the duration of cooling process. But overall, it is hard to identify the optimal conditions to follow.

Many health experts suggest continuous (standard) protocol that maintains cooling for a continuous period of time; like 20 minutes of continuous icing every two hours. This protocol is 
widely used in clinical settings $[2,3]$. On the other side, there is another group that prefers intermittent protocol which applies cooling for a discrete periods of time and removes cooling between these discrete intervals (like 10 minutes on 10 minutes off).

For this project continuous icing is the proposed protocol but in the upcoming days, the analysis will be done again using intermitted protocol to compare both techniques and to get a clearer vision of these two protocols.

\section{B. Design Objectives}

Beside the clear vision for heat flow within the system that could be offered by software like COMSOL Multiphysics, there are also many goals that are hoped to be achieved in this research which are represented in the following:

- Helping to find the optimal temperature and periods of cryotherapy treatment and identifying the more effective protocol. As well as keeping the temperature within the therapeutic range, and at the same time avoiding pain and tissue damage.

- Analyzing the efficiency of the ice packing and the other suggested layers, by showing the heat flow within each layer.

- Examine the effectiveness of using materials like thermoplastic, hydrogel, and cotton in thermal applications.

- Modifying and determining the best combination and arrangement of the materials used in these layers to achieve the desired cooling within the required ranges.

- $\quad$ Protecting the patient from tissue necrosis that could be occurs due improper use of cooling.

- Offering an imagination for the ideal biobandage design for burns and thermal applications.

Beside all these goals, the study has many inclusions, the arranged information in this research paper can be very helpful to understand the burns and the physiological activities of the human body in case of burn, it also offer better understanding for the thermal properties of the materials, heat flow governing equations and variables of these governing equation, and how these variables relate to each other in mathematical calculations. This could be a very well organized information base to any future work in this field.

\section{MATHEMATICAL STATEMENT}

\section{A. Geometry Development}

The main aim of this design is to achieve perfect cooling to the burned tissue which in turn makes the healing process faster, so, choosing appropriate geometries is the first step toward this goal.

The design is composed of four layers, the ice layer, thermoplastic layer, water gel layer and cotton layer. The ice layer will be the upper outer layer, while the cotton layer will be the one in direct contact with the burned tissue. For the tissue, it is better to consider it as three main layers, skin, fat and muscle layer, but in this analysis only skin will be considered.

The geometry is modeled as two-dimensional but for simplicity, the analysis are performed as a one-dimensional, this can normally be assumed as the main interest is approximately the vertical heat flow on the skin.

It is also assumed that the ice volume is not changing as it melts to form water. This assumption is based on the implication that the ice is fully packed and the water that will form remains in the pack.

A rectangular shape was chosen for the layers while, thickness of each layer was chosen approximately, but after getting the results, it can be easily modified to get the desired cooling. Skin is also considered as a layer with similar width of the bandage as it is the actual area of interest, but it has a specific thickness.

Although, skin thickness differs from burn to burn according to the type and depth, in this analysis it is considered to be $3 \mathrm{~mm}$. Maybe in future work, the analysis will be repeated for the four types of burns with a specific thickness for each type based on the degree of damaging. The detailed dimensions for the layers are as shown in table 1 below.

Table 1: Dimensions of bandage layers

\begin{tabular}{|l|c|}
\hline Layer & $\begin{array}{l}\text { Thickness (height) } \\
(\mathrm{mm})\end{array}$ \\
\hline Ice Layer & 4 \\
\hline Thermoplastic & 1 \\
\hline Hydrogel & 1 \\
\hline Cotton & 1 \\
\hline Skin & 3 \\
\hline
\end{tabular}

The computational domain for the bandage and the tissue is divided into five domains as shown in Figure 1, each layer treated as a separated domain with its different properties and specifications.

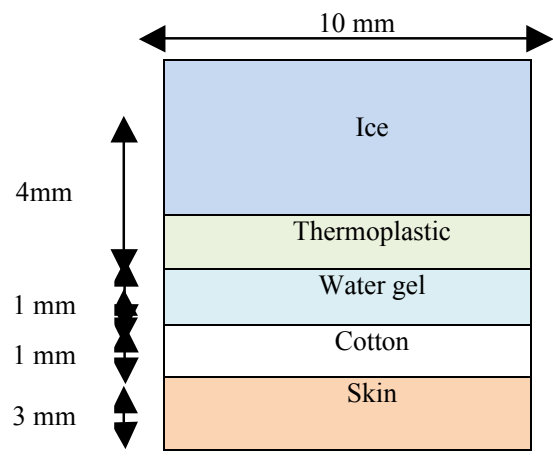

Figure1: Computational domain of bandage layers

\section{B. Design Assumptions}

This analysis is considered as a first step for more detailed studies in the future, so, many assumptions were implemented to simplify this model. 
Although the system is more complicated than our assumptions as a foundation it would be reasonable to do simulation for a general cooling model.

The bandage was modeled as vertical series rectangles of various height (thickness) over each other, each rectangle represent a layer of the bandage.

Skin is mainly composed of three layers, epidermis, dermis, and subcutaneous fat layer, these layers have different thermal properties but it is not that much difference, so for more simplicity these three layers will be combined in one layer and modeled as one domain. A weighted average of physical and thermal properties of these three layers together was taken to be used for the skin overall.

For muscles and bones, it can be assumed that they are with skin as one homogeneous material, but in this analysis they are not of interest.

\section{Selection of physical and thermal properties}

Material properties are important issue, as these properties will directly affect the heat flow within the layers of the design, and overall heat exchanging as well.

In this type of analysis, the main interest is with the thermal properties of the material, that's why choosing the materials is basically based on these properties, specially, thermal conductivity and specific heat capacity of the material. Density of the material is also important and should be taken into account in these calculations.

The main physical and thermal properties for the skin and the other materials used in this model are shown in (table 2) [4].

Table 2: Physical and thermal properties of the materials [4]

\begin{tabular}{|l|c|c|c|}
\hline \multicolumn{1}{|c|}{ material } & $\begin{array}{c}\text { Thermal } \\
\text { Conductivity }(k) \\
\mathrm{W} / \mathrm{m} \cdot \mathrm{K}\end{array}$ & $\begin{array}{c}\text { Density }(\rho) \\
\mathrm{kg} / \mathrm{m}^{3}\end{array}$ & $\begin{array}{c}\text { Specific Heat } \\
\text { Capacity }(C p) \\
\mathrm{J} / \mathrm{kg} \cdot \mathrm{K}\end{array}$ \\
\hline Ice & 1.88 & 920 & 2100 \\
\hline Thermoplastic & 0.2 & 1200 & 1200 \\
\hline Hydrogel & 0.258 & 1097 & 2360 \\
\hline Cotton & 0.04 & 200 & 1340 \\
\hline Skin & 0.37 & 1109 & 3391 \\
\hline
\end{tabular}

The study was performed as a time dependent study to examine the changing of temperature with the time.

\section{Governing equations}

The general form of governing equations for a timedependent heat transfer in the bandage and skin system along the depth is[5]:

$$
\rho C p \frac{\partial T}{\partial t}+\rho C p u * \nabla T=\nabla *(k \nabla T)+Q
$$

Where $\rho$ is the density of the material, $C p$ is the specific heat capacity, $k$ is the thermal conductivity, and Q is the term for bioheat.
Pennes [6] dealt with blood perfusion rate which plays a significant role in metabolic rate and cells heat production. He assumed that for any small volume of tissue, blood temperatures due the entering and exiting of the capillary are constant. In other words that means that blood temperatures (arterial temperature) at any region of the tissue are the same as the local tissue temperature (the surrounding).

$$
Q=q_{m}+q_{p}
$$

Where $q_{p}$ is the term for perfusion heat source $\left[\mathrm{W} / \mathrm{m}^{3}\right], q_{m}$ is the metabolic heat source $\left[\mathrm{W} / \mathrm{m}^{3}\right]$, and $\mathrm{k}$ is thermal conductivity.

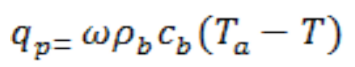

where, $\omega$ is the blood perfusion rate $[\mathrm{ml} / 100 \mathrm{ml}-\mathrm{s}], \rho_{b}$ the density of the blood $\left[\mathrm{kg} / \mathrm{m}^{3}\right], \quad C_{b}$ is the specific heat capacity of the blood $[\mathrm{J} / \mathrm{kgK}]$, and, $\left(T_{a}-T\right)$ is the difference between arterial blood temperature and local tissue temperature.

With these three equations it is easily recognized the effect of any variable on the total heat flux, but in this project and as there is no enough time to do all these analysis, the model will not include the metabolic and blood perfusion effect but they will be included theoretically in analysis process, calculations and discussion.

\section{E. Initial and boundary conditions}

The initial and boundary conditions are an important issues in any molding analysis, and they vary due to different cooling situations that requires assuming a correct values inputs to approximately reflect the real case.

Figure 2 shows the boundary conditions and initial conditions for this model. The upper surface of the ice which is the top external boundary layer of the overall bandage is assumed to be thermally insulated. Zero flux is also assumed for both external boundaries (sides) of all the layers to convert a two dimensional heat transfer problem to one-dimensional heat transfer along the thickness. The bottom of the skin surface is assumed to be at $323.15 \mathrm{~K}$.

The initial temperature for the bandage is $273.15 \mathrm{~K}$. Temperature of skin varies according to the burns degree and type, mostly for burned tissue, it is around $45-50^{\circ} \mathrm{C}$. In this analysis it was considered $50^{\circ} \mathrm{C}$ which equals $323.15 \mathrm{~K}$.

Usually water gel layer is considered as a cooling source like ice and has low temperatures mostly, around zero temperature, but initially, it will be taken as a normal layer and according to the achieved results its temperature can be modified to get a desired cooling. 
$\mathrm{T}_{\mathrm{n}}=273.15 \mathrm{~K}$

$\mathrm{T}_{\mathrm{o}}=323.15 \mathrm{~K}$

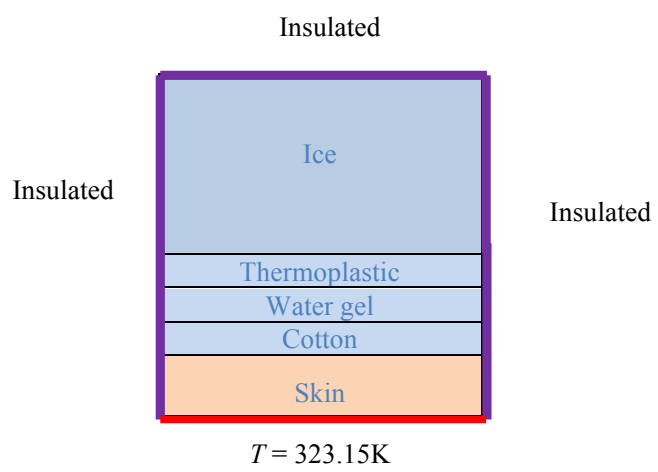

Figure 2: Initial and boundary conditions input for bandage model

\section{F. Numerical method}

A time-dependent heat transfer problem defined above was solved in a FEM software package COMSOL to study the 20 minutes cooling process of an iced bandage placed on a burn. A free triangular meshing as shown in Fig. 3 was used to mesh the computational domain. Finer meshes are generated near the interfaces between different layers. Mesh convergence study has taken to choose an appropriate set of mesh for further study.

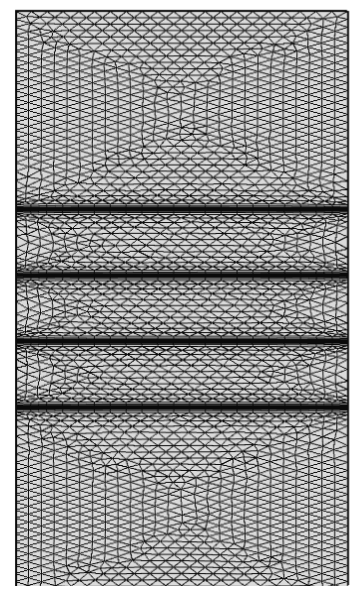

Figure 3: Mesh of the model.

\section{RESULTS AND DISCUSSION}

\section{A. Temperture evolution inside the whole domain}

Figure 4 shows the temperature field in the whole computational domain which includes the four layers in bandage and the skin layer at various time steps. It can be seen that the temperature in the skin layer is lowered from the initial $323.15 \mathrm{~K}$ temperature during the cooling process. Heat is transfer from the burn upward to the bandage above. The temperature rise inside the cotton layer, water gel layer and thermoplastic layer is faster compared to the temperature in the ice layer. The detailed temperature response can be clearly seen in the temperature profiles along the thickness of the computational domain at various time steps as shown in Fig. 5 .

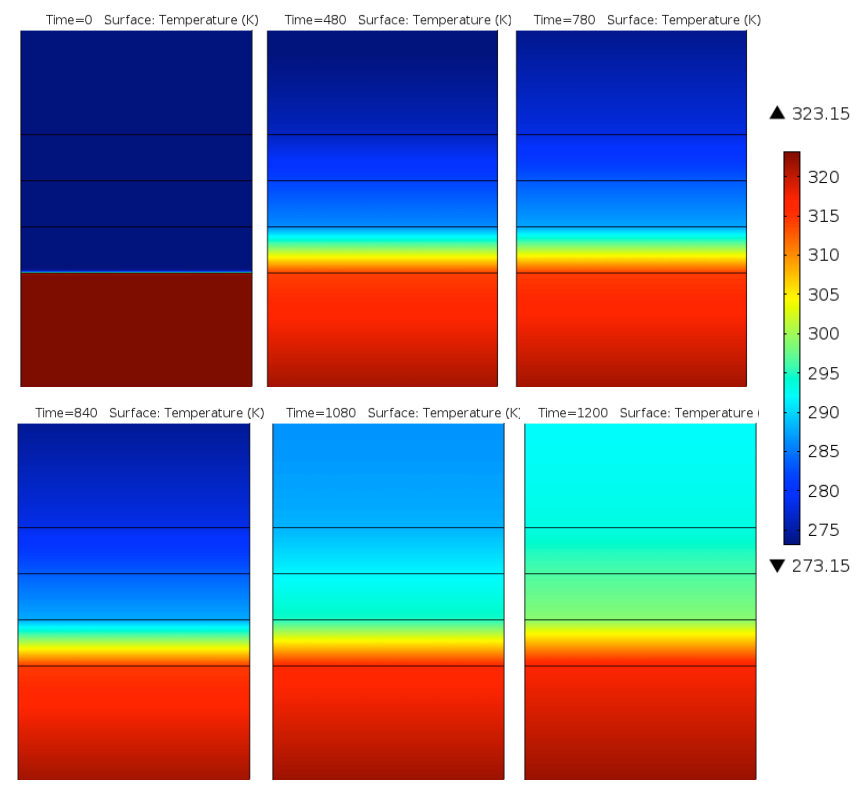

Figure 4: Temperature field in bandage and skin at various time steps.

Figure 5 (a) shows the temperature profiles along the thickness direction at various time instants within the first minute of cooling. It can be seen that the temperature profiles show the gradual cooling in the skin layer and temperature rise in the cotton layer, water gel layer and the thermoplastic layer. The temperature in the ice layer is mainly intact. The temperature profiles have remarkable changes within the $30 \mathrm{~s}$ and then the changes decreased in the next 30s. The whole system reaches a quasi-steady state within a minute. The cotton layer has the fastest response to the temperature change and also has the highest temperature drop among the five layers. The fastest response is due to its low thermal capacity and low density and the high temperature drop is caused by its low thermal conductivity. The thermal physical properties of skin are close to those of water gel and thermal plastic, thus similar temperature profiles are found in those layers.

The entire system maintains a quasi-steady state between until around 840s as shown in Fig. 5 (b). During this period, the temperature in the skin layer has no significant change and the temperature in the ice layer gradually increase. The amplified temperature profiles in the ice layer shown Fig. 6 suggest that the ice layer is melt during this period and the phase change completes at around $800 \mathrm{~s}$.

After the ice in the bandage completely changes to water, temperature in the ice layer starts to increase and thus drives up the temperature in the system to increase, as shown in Fig. $5(\mathrm{c})$. 


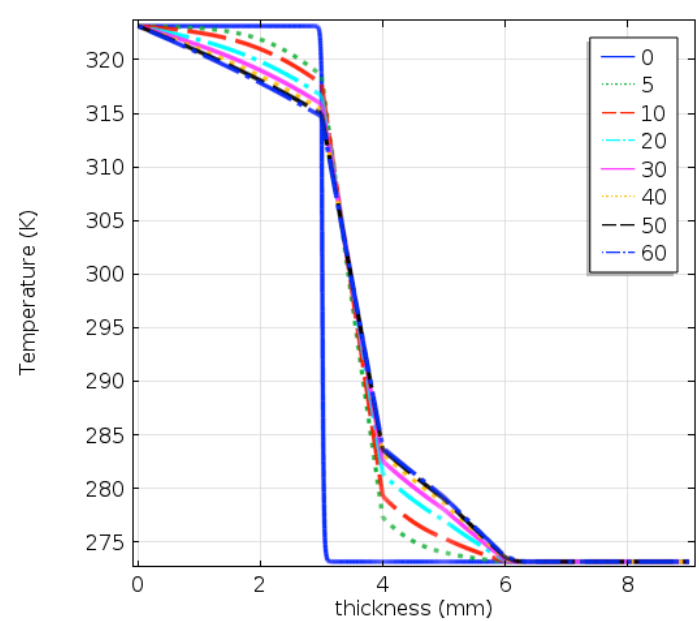

(a)

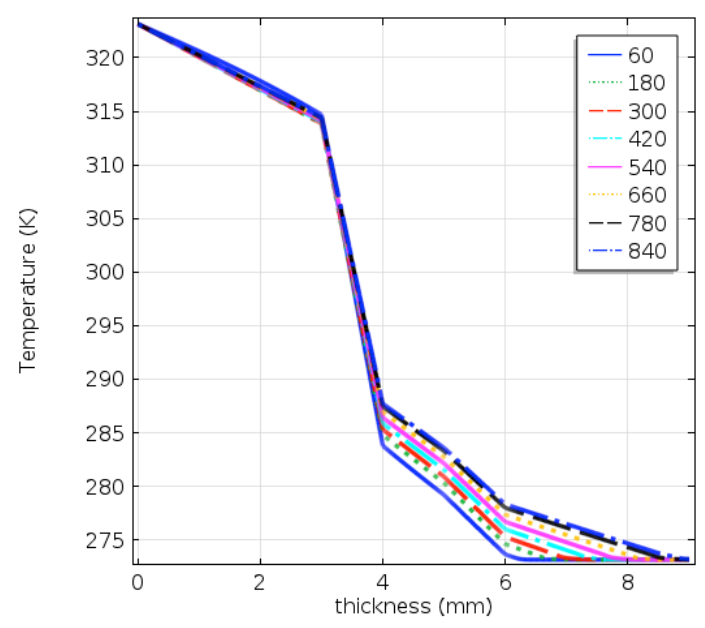

(b)

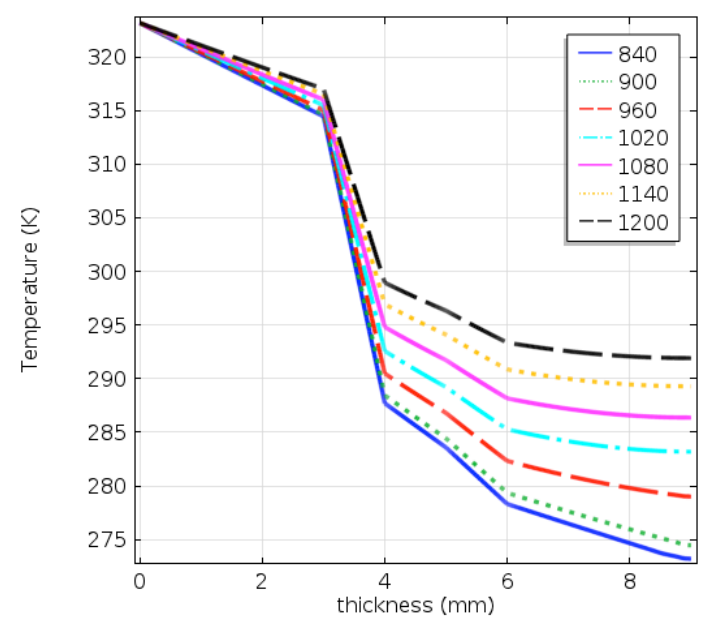

(c)

Figure 5: Temperature profiles along the thickness of the computational domain various time steps: (a) from $0 \mathrm{~s}$ to $60 \mathrm{~s}$, (b) from $60 \mathrm{~s}$ to $780 \mathrm{~s}$, and (c) from 780 s to 1200 s.

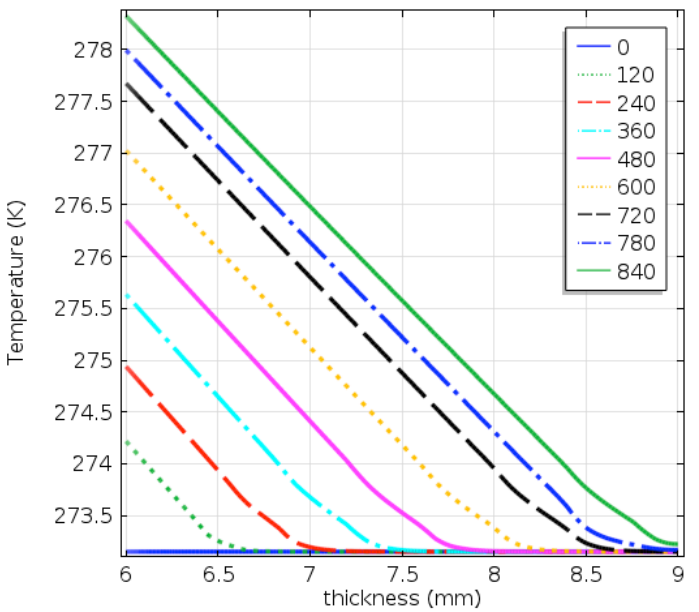

Figure 6: Temperature profiles of ice from 0 s to $840 \mathrm{~s}$.

\section{B. Temperture history and heat flux at the skin surface}

Figure 7 show the temperature history at the skin surface. It shows a quick temperature drop in the first minute. The temperature reaches its minimum of $314^{\circ} \mathrm{C}$ at $155 \mathrm{~s}$. After reaches the bottom, it continues to rise slowly until reaches 860 s when it starts a fast increase. The temperature evolution at the skin surface can be further explained with the temperature profiles in the skin layer shown in Fig. 8. The temperature profiles during the cooling process as shown in Fig. 8(a) shows the response of the skin layer cooled by a cold iced package. The minimum temperature at the skin would be a very import indicator for a safe and effective cooling. As we see in Fig. 5 (b) that the highest temperature drop is in the cotton layer, the most effective way to design a bandage for a desired temperature is to adjust the thickness of the cotton layer. The temperature profiles in the skin layer in Fig. 8(b) show the slowly temperature rise process from 180s to 900 s and then a faster rise after the ice layer completely changes to water.

As it can be seen in Fig. 9, the history of heat flux at the skin surface shows the reverse pattern of its temperature history. The high heat flux in the first few minutes drives the temperature at the skin surface to the minimum and then a steady slow decrease of heat flux as ice is melt till all the ice are melted. As ice absorbs heat without increasing temperature when it changes to water, the ice melting process stabilizes the cooling process. After the ice is depleted, water absorbs heat by increasing its temperature and it in turn lower the heat absorb capacity of the system. As a result, the heat flux decreases with steeper slope. 


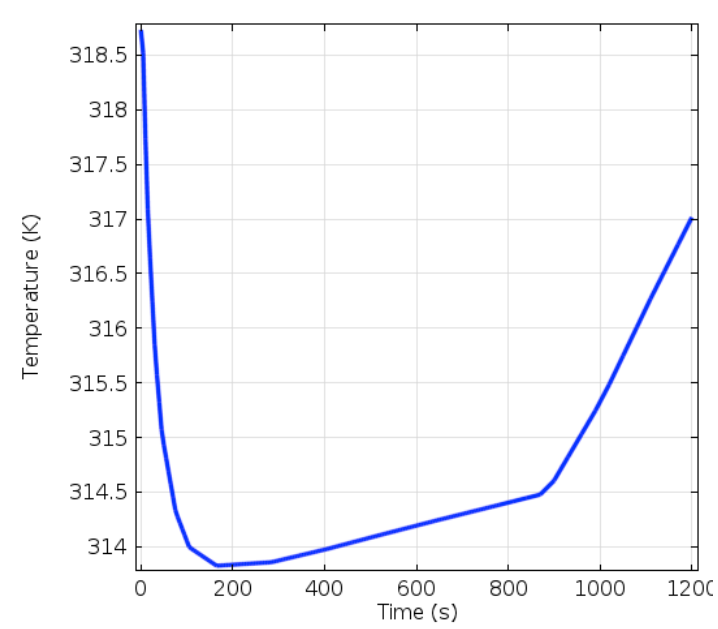

Figure 7: Temperature history at the skin surface

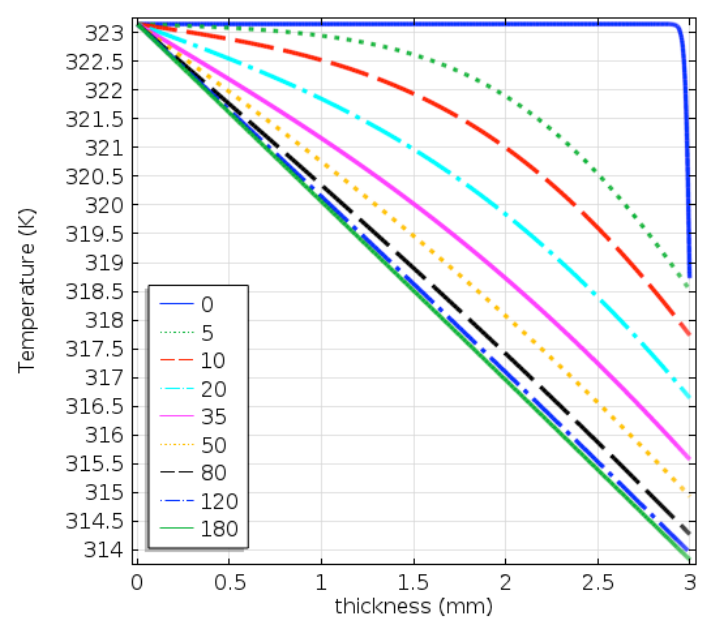

(a)

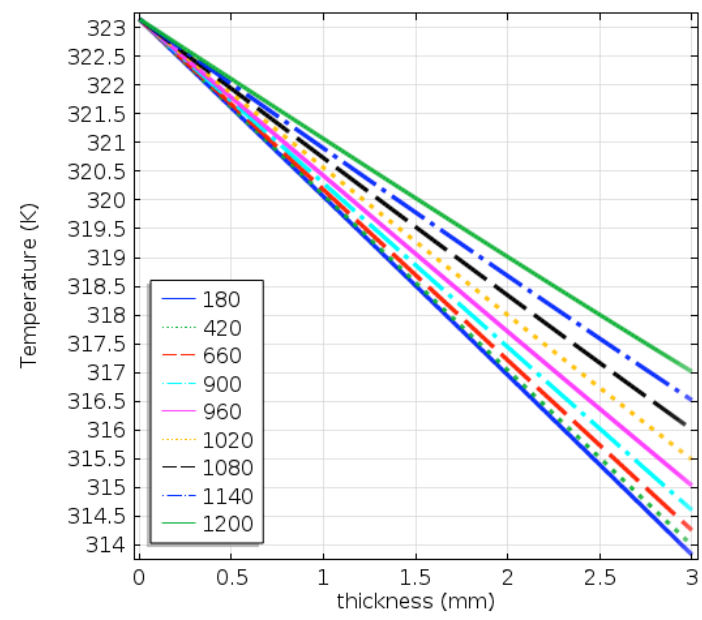

(b)

Figure 8: Temperature profile evolution in the skin layer along the depth: (a) from $0 \mathrm{~s}$ to $180 \mathrm{~s}$ and (b) from $180 \mathrm{~s}$ to $1200 \mathrm{~s}$

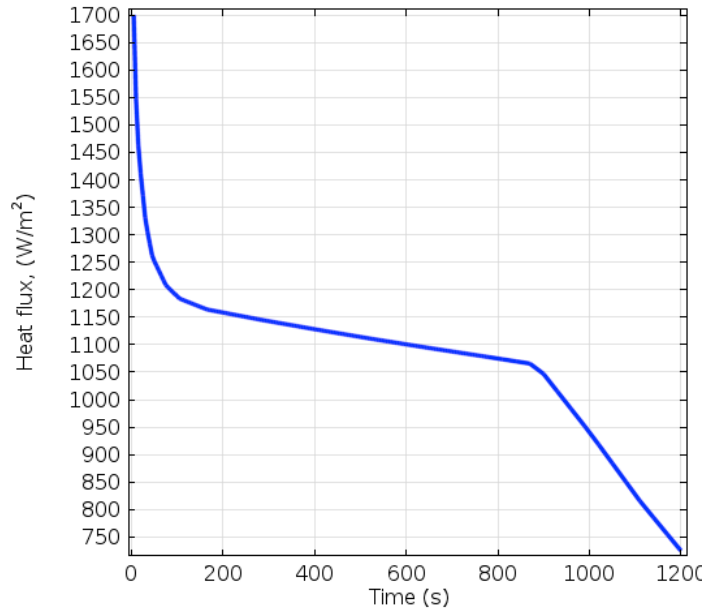

Figure 9: Heat flux history at the skin surface

As shown from the previous figures, we can recognize that the effective cooling process happened during the first 14 minutes as the temperature of the skin droped from $323 \mathrm{~K}$ to $314.5 \mathrm{~K}$ (about 4.5 degrees of the normal body temperature) which is considered a highly beneficial change in the physiological system.

This somehow leads us to the optimal period of topical ice cooling that should be used for continuous (standard) protocol, as the ice layer is melted during this period and the phase change completes at around 800 s, then the temperature begins a faster rise after the ice layer completely changes to water. That means, after 14 minutes the ice layer is no longer effective, and can be removed and replaced with another layer. The interval bewteen two iced bandages can be optimized for different cooling protocol.

\section{CONCLUSION}

Magnificent effect could be achieved by cooling, but any cooling process should be performed carefully within the right procedure and the right optimal temperature. The temperature must be kept within the therapeutic range, to avoid any side effects or tissue damage (tissue necrosis), and at the same time avoiding pain and providing a comfort healing process.

More analysis needed to be done to find the optimal temperature and periods of cryotherapy treatment which helps us to identify the more effective protocol.

Not only is the ice layer important but also the other layers of the biobandage which should be chosen carefully according to their thermal properties to achieve this goal. The combination of all the chosen layers are physically working together to reduce inflammation. Lowering the temperature makes the heeling or recovery time shorter and decreases the nerve conductivity which in turn reduces pain as well.

The thickness of the layers is important, and it can be modified to get the desired results. The model overall can also be easily modified and many combinations of materials can be used for this purpose 
The study has many inclusions, the arranged information in this project can be very helpful to understand the burns and the physiological activities of the human body in case of burn, it also offer better understanding for the thermal properties of the materials, heat flow governing equations and variables of these governing equation, and how these variables relate to each other in mathematical calculations. This could be a very well organized information base to any future work in this field

\section{RECOMMENDATIONS AND FUTURE WORK}

- In the future researchers should carefully analyze the effect of the metabolic rate and blood perfusion on the mathematics of the governing equation.

- Remodel this idea for each degree of burns taking into account the specific thickness of the burned tissue and the size of the tissue damage.

- Look to analyze other combinations of materials such as Poly-lactic acid.

- Using the water-gel layer in a more effective way, as it could be as effective as the ice layer

- Examine the intermitted protocol and make detailed comparisons between both protocols

\section{REFERENCES}

[1] Weber, Kathy. "The technical benefits of icing." Moji. 2009. http://www.gomoji.com/education/technical-benefits-icing

[2] Swenson C, Sward L, Karlsson J. "Cryotherapy in sports medicine." Scand J Med Sci Sports 1996; 6:193-200.

[3] Kerr KM, Daily L, Booth L. Guidelines for the management of soft tissue (musculoskeletal) injury with protection, rest, ice, compression and elevation

(PRICE) during the first 64 hours. London: Chartered Society of Physiotherapy, 1999.

[4] www.Engineeringtoolbox.com

[5] Tu J, Yeoh GH, Liu C, (2012). Computational Fluid Dynamics: A Practical Approach, 2nd Edition. Butterworth-Heinemann. ISBN-10: 0080982433.

[6] E.H. Wissler. "Pennes; 1948 Paper Revisited."Journal of Applied Physiology. July 1998 vol. 85, no. $135-41$.

[7] van de Staak WJBM, Brakkee AJM, de Rijke-Herweijer HE: Measurements of the thermal conductivity of the skin as an indication of skin blood flow. J Invest Dermatol 1968 51: 149-154

[8] Datta A, Rakesh V, (2009). An Introduction to Modeling of Transport Processes: Application to Biomedical Systems, $1^{\text {st }}$ Edition. Cambridge University Press. ISBN-10:0521119243

[9] Smith JJ and Fraser J. An estimation of tissue damage and thermal history in the cryolesion. Cryobiology 11 (1974), pp. 139-147.

[10] Rossi MR, Tanaka D, Shimada K, Rabin Y. An efficient numerical technique for bioheat simulations and its application to computerized cryosurgery planning. Computer methods and programs in biomedicine. 85 (2007), pp.41-50.

[11] Rewcastle JC, Sandison GA, Hahn LJ, Saliken JC, McKinnon JG, Donnelley BJ. A model for the time-dependent thermal distribution within an ice ball surrounding a cryoprobe. Phys. Med. Biol. 43 (1998), pp. 3519-3534.

[12] Gage A, Baust J. Mechanisms of tissue injury in cryosurgery. Cryobiology 37(3) (1998), pp. 171-186. 